\title{
Clinical differences between bipolar and unipolar depression
}

Liz Forty, Daniel Smith, Lisa Jones, Ian Jones, Sian Caesar, Carly Cooper, Christine Fraser, Katherine Gordon-Smith, Sally Hyde, Anne Farmer, Peter McGuffin and Nick Craddock

\section{Summary}

It is commonly - but wrongly - assumed that there are no important differences between the clinical presentations of major depressive disorder and bipolar depression. Here we compare clinical course variables and depressive symptom profiles in a large sample of individuals with major depressive disorder $(n=593)$ and bipolar disorder $(n=443)$. Clinical characteristics associated with a bipolar course included the presence of psychosis, diurnal mood variation and hypersomnia during depressive episodes, and a greater number of shorter depressive episodes. Such features should alert a clinician to a possible bipolar course. This is important because optimal management is not the same for bipolar and unipolar depression.

\section{Declaration of interest}

Funding from the Wellcome Trust, the UK Medical Research Council and GlaxosmithKline.
Distinguishing between major depressive disorder and bipolar disorder is important because there are differences in the optimal management of these conditions. Antidepressant treatment of bipolar depression can adversely affect long-term prognosis by causing destabilisation of mood and more frequent depressive episodes, and can lead to the development of treatment resistance. ${ }^{1}$ Most people with bipolar disorder experience depression rather than mania as their first episode of illness. It is clinically desirable to recognise, or at least to suspect, bipolar depression at an early stage of a bipolar illness. Here, we compare the clinical course and depressive symptoms of the two forms of depression by analysing phenotypic data for over 1000 patients who were recruited to our genetic epidemiological studies of mood disorders.

\section{Method}

The sample comprised 443 individuals with type I bipolar disorder and 593 with recurrent major depressive disorder. Participants were recruited systematically from the case-loads of community mental health teams, and non-systematically from advertisements placed in local general practices and local media. Patients were excluded from the original genetic studies if they: had a lifetime diagnosis of injecting drug dependency; had only experienced affective illness as a result of alcohol or substance dependence; had only experienced affective illness secondary to medical illness or medication; or were biologically related to another study participant. In addition, people with major depressive disorder were excluded if they had a first- or second-degree relative with a clear diagnosis of bipolar disorder or schizophrenia, schizotypal disorder, persistent delusional disorder, acute and transient psychotic disorders or schizoaffective disorder, or had ever experienced mood-incongruent psychosis or psychosis outside of mood episodes.

Participants completed the Beck Depression Inventory (BDI) ${ }^{2}$ and were interviewed using the Schedules for Clinical Assessment in Neuropsychiatry (SCAN), ${ }^{3}$ which provides detailed information about lifetime psychopathology. Psychiatric and general practice case notes were reviewed. These data were combined to form a written case vignette. Based on this vignette, best-estimate lifetime diagnoses were made according to DSM-IV criteria. ${ }^{4}$ Vignettes were also used to rate key clinical variables (such as age at onset, and number and severity of episodes of illness). The operational criteria (OPCRIT) symptom checklist was used to rate presence or absence of depressive, manic and psychotic symptoms on a lifetime-ever basis. ${ }^{5,6}$ Each participant was diagnosed and had key clinical variables rated independently by at least two members of the research team, and a consensus was reached. Team members involved in the interview, rating and diagnostic procedures were either fully trained research psychologists or psychiatrists. Interrater reliability was assessed using joint ratings of 20 cases with a range of mood disorder diagnoses. Mean overall kappa was 0.85 for DSM-IV diagnoses. Mean kappa statistics and intraclass correlation coefficients for other key clinical variables were in the range $0.81-0.99$ and $0.85-0.97$ respectively. This study received all necessary multiregion and local research ethics committee approval, and all participants gave written informed consent.

Forward stepwise likelihood ratio binary logistic regression was performed using the Statistical Package for the Social Sciences, SPSS version 12 for Windows, to identify variables significantly predictive of bipolar disorder $v$. major depressive disorder classification.

\section{Results}

The proportions of women in the major depression group and the bipolar group were $70.2 \%$ and $71.3 \%$ respectively. The median age at interview was 49 years for the major depression group and 47 years for the bipolar group. Forty-six per cent of the major depression group were recruited systematically, compared with $37 \%$ of the bipolar group. The median illness duration was 19 years for the major depression group and 20 years for the bipolar group. The major depression group had a median BDI score at interview of 16 , compared with 8 in the bipolar group.

Forward stepwise logistic regression was used to establish the best depression-related predictors of bipolar $v$. unipolar group membership. All lifetime variables relating to depressive episodes that were significant at the $1 \%$ level in univariate analyses comparing the two groups were entered into the regression. To control for sample differences in recruitment and current mental state, BDI score at interview and method of recruitment were included in the regression. Gender was also included in the logistic regression analysis. Significant predictors of diagnosis are shown in Table 1.

Although there were, of course, similarities between unipolar and bipolar depression, we found important clinical differences: characteristics that best predicted bipolar rather than unipolar 
Table 1 Lifetime clinical characteristics predicting bipolar v. unipolar group membership

\begin{tabular}{|c|c|c|c|c|}
\hline & $\begin{array}{l}\text { Major depressive } \\
\text { disorder }\end{array}$ & Bipolar disorder & OR $(95 \% \mathrm{Cl})^{\mathrm{a}}$ & $P$ \\
\hline Participants, $n$ (total $n=1036$ ) & 593 & 443 & & \\
\hline Psychotic features during depression, $n(\%)^{\mathrm{b}}$ : present/absent & $61(10.5) / 522(89.5)$ & $134(30.2) / 309(69.8)$ & $0.160(0.08-0.32)$ & $<0.001$ \\
\hline No. of episodes of depression: median (IQR) range & 4 (2) 2-40 & $5(6) 1-70$ & $0.932(0.89-0.98)$ & 0.006 \\
\hline Longest episode of depression, weeks: median (IQR) range & $69(60) 8-624$ & $26(29) 2-416$ & $1.011(1.01-1.02)$ & $<0.001$ \\
\hline Diurnal mood variation, $n(\%)^{\mathrm{b}}$ : present/absent & $285(50.4) / 281(49.6)$ & $219(59) / 152(41)$ & $0.536(0.31-0.94)$ & 0.030 \\
\hline Excessive self-reproach, $n(\%)^{\mathrm{b}}$ : present/absent & $550(96.2) / 22(3.8)$ & $342(87.7) / 48(12.3)$ & $6.272(2.34-16.85)$ & 0.000 \\
\hline Loss of energy, $n(\%)^{\mathrm{b}}:$ present/absent & $584(99.2) / 5(0.8)$ & 386 (95.5)/18 (4.5) & $6.031(1.00-36.27)$ & 0.050 \\
\hline Hypersomnia, $n(\%)^{\mathrm{b}}$ : present/absent & $120(21.5) / 437(78.5)$ & $148(42.8) / 198(57.2)$ & $0.371(0.21-0.67)$ & 0.001 \\
\hline Diminished libido, $n(\%)^{\mathrm{b}}$ : present/absent & $231(63.5) / 133(36.5)$ & $123(34.8) / 230(65.2)$ & $7.537(4.14-13.74)$ & $<0.001$ \\
\hline \multicolumn{5}{|c|}{$\begin{array}{l}\text { IQR, interquartile range. } \\
\text { a. The odds ratios (ORS) shown are from a logistic regression analysis predicting bipolar or unipolar group membership according to lifetime clinical characteristics. With the coding } \\
\text { used, OR } 1 \text { indicates that a higher score is associated with greater likelihood of unipolar group membership, whereas OR }<1 \text { indicates that a higher score is associated with a } \\
\text { greater likelihood of bipolar group membership. } \\
\text { b. values of } n \text { may vary due to missing/unclear data. }\end{array}$} \\
\hline
\end{tabular}

depression were the presence of psychosis, diurnal mood variation and hypersomnia during depressive episodes, a greater number of depressive episodes and a shorter duration of the longest depressive episode. Participants with major depressive disorder were characterised by the presence of excessive self-reproach, loss of energy and diminished libido.

\section{Discussion}

Our results are consistent with, and extend the findings of, previous studies that have shown that 'atypical' depressive features (such as hypersomnia and weight gain) may be more common in bipolar disorder than in major depression. ${ }^{7-9}$ Compared with previous studies, our study has several advantages, including the large number of participants and the high degree of consistent and comprehensive clinical data collected.

Distinguishing between bipolar disorder and major depressive disorder is of great clinical importance because optimal management of the two conditions is very different. For example, antidepressants should be used with caution in bipolar depression because of the risk of precipitating mood switches, cycling, or mixed or agitated states. ${ }^{10}$ It is desirable that clinicians use all available information to guide management (including choice of treatment, advice to patient and intensity of monitoring). The clinical features of depression are not a definitive guide to diagnosis but can help to alert the clinician to a possible bipolar course. These findings also have important implications for future research on type II bipolar disorder and sub-threshold bipolar disorders. Evidence suggests that $25-50 \%$ of individuals with recurrent major depression (particularly those within atypical, early-onset or treatment-refractory subgroups) may in fact have a broadly defined bipolar disorder. ${ }^{11}$ We currently know little about how best to treat such patients. Future studies will need to move beyond strict diagnostic categories and examine subgroups of patients defined by extended phenotypic measures such as dimensional assessments of bipolar features, bipolar symptom clusters and longitudinal illness course variables.

An important limitation of our study is that there might have been differences between the two groups of participants that we were not able to examine, such as subtle differences in treatment regimens or patterns of comorbid illness. We also note that although the proportion of women in the major depressive disorder sample is typical of studies of this nature, the proportion of women in the bipolar disorder sample is higher than is typically reported (nearly three-quarters compared with a half) and that this may limit the generalisability of our findings. A further limitation was the use of retrospective rather than prospective assessments, even though we used an in-depth semi-structured clinical interview supplemented by case-note review. Prospective ratings, though preferable, can be prohibitively expensive.

Liz Forty, BSC, Daniel Smith, MD, Department of Psychological Medicine, School of Medicine, Cardiff University; Lisa Jones, PhD, Department of Psychiatry, Division of Neuroscience, University of Birmingham; Ian Jones, PhD, Department of Psychological Medicine, School of Medicine, Cardiff University; Sian Caesar, BSc, Carly Cooper, BSC, Department of Psychiatry, Division of Neuroscience, University of Birmingham; Christine Fraser, BSC, Katherine Gordon-Smith, PhD, Department of Birmingham; Christine Fraser, BSC, Katherine Gordon-Smith, PhD, Department
Psychological Medicine, School of Medicine, Cardiff University; Sally Hyde, BSc, Department of Psychiatry, Division of Neuroscience, University of Birmingham; Anne Farmer, MD, Peter McGuffin, PhD, Medical Research Council Social, Genetic and Developmental Psychiatry Centre, Institute of Psychiatry, London; Nick Craddock, PhD, Department of Psychological Medicine, School of Medicine, Cardiff University, Cardiff

Correspondence: Professor Nick Craddock, Department of Psychological Medicine, Henry Wellcome Building, Wales School of Medicine, Cardiff University, Heath Park, Cardiff CF14 4XN, UK. Email: craddockn@cf.ac.uk

First received 18 Sep 2007, final revision 17 Jan 2008, accepted 24 Jan 2008

\section{References}

1 Sharma V, Khan M, Smith A. A closer look at treament resistant depression: is it due to a bipolar diathesis? J Affect Disord 2005; 84: 251-7.

2 Beck A, Steer R. The Beck Depression Inventory. Harcourt Brace, 1987.

3 Wing J, Babor M, Brugha T, Burke J, Cooper J, Giel R, Jablenski A, Regier D. SCAN: Schedules for Clinical Assessment in Neuropsychiatry. Arch Gen Psychiatry 1990; 47: 589-93.

4 American Psychiatric Association. Diagnostic and Statistical Manual of Mental Disorders (4th edn) (DSM-IV). APA, 1994.

5 McGuffin P, Farmer A, Harvey I. A polydiagnostic application of operational criteria in studies of psychotic illness. Development and reliability of the OPCRIT system. Arch Gen Psychiatry 1991; 48: 764-70.

6 Craddock M, Asherson P, Owen MJ, Williams J, McGuffin P, Farmer AE. Concurrent validity of the OPCRIT diagnostic system. Comparison of OPCRIT diagnoses with consensus best-estimate lifetime diagnoses. Br J Psychiatry 1996; 169: 58-63.

7 Bowden CL. A different depression: clinical distinctions between bipolar and unipolar depression. J Affect Disord 2005; 84: 117-25.

8 Swann AC, Geller B, Post RM, Altshuler L, Chang KD, Delbello MP, Reist C, Juster IA. Practical clues to early recognition of bipolar disorder: a primary care approach. Prim Care Companion J Clin Psychiatry 2005; 7: 15-21.

9 Mitchell PB, Wilhelm K, Parker G, Austin MP, Rutgers P, Malhi GS. The clinical features of bipolar depression: a comparison with matched major depressive disorder patients. J Clin Psychiatry 2001; 62: 212-16.

10 National Institute for Health and Clinical Excellence. The Management of Bipolar Disorder in Adults, Children and Adolescents, in Primary and Secondary Care. Clinical Guideline 38. NICE, 2006.

11 Angst J. The bipolar spectrum. Br J Psychiatry 2007; 190: 189-91. 